\title{
Alessandro Mariani, La deconstruzione in pedagogia. Una frontiera teorico-educativa della postmodernità
}

Rome, Armando Editore, 2008, 144 p.

Michel Ostenc

\section{OpenEdition}

Journals

Édition électronique

URL : http://journals.openedition.org/assr/22917

DOI : $10.4000 /$ assr.22917

ISSN : $1777-5825$

Éditeur

Éditions de l'EHESS

Édition imprimée

Date de publication : 31 décembre 2011

Pagination : 216

ISBN : 9782713223273

ISSN : 0335-5985

\section{Référence électronique}

Michel Ostenc, «Alessandro Mariani, La deconstruzione in pedagogia. Una frontiera teorico-educativa della postmodernità », Archives de sciences sociales des religions [En ligne], 156 | octobre-décembre 2011, document 156-75, mis en ligne le 16 février 2012, consulté le 21 septembre 2020. URL : http:// journals.openedition.org/assr/22917 ; DOI : https://doi.org/10.4000/assr.22917 


\title{
Alessandro Mariani, La deconstruzione in pedagogia. Una frontiera teorico-educativa della postmodernità
}

Rome, Armando Editore, 2008, 144 p.

\author{
Michel Ostenc
}

\section{RÉFÉRENCE}

Alessandro MARIANI, La deconstruzione in pedagogia. Una frontiera teorico-educativa della postmodernità, Rome, Armando Editore, 2008, $144 \mathrm{p}$.

1 L'auteur situe les références philosophiques de la pensée de Jacques Derrida chez Nietzsche et Heidegger, aussi bien que chez Freud, Lacan et Levinas. Ce sont des réflexions sur les discontinuités historiques et scientifiques, sur le caractère subjectif de l'«expérience de l'être » et sur son incapacité à représenter la seule instance de l'identité, sur une psychologie enfin où la prise de conscience de l'humanité de l'autre précède celle du sujet lui-même. En fait, Derrida fait un choix dans cet héritage qu'il entend soumettre à la critique de la « déconstruction ». Le point de départ se situe dans une phénoménologie qui fait abstraction des préjugés culturels et des évidences de la perception pour parvenir à une vision plus objective du monde ; mais Derrida ne croit pas à l'efficacité d'une méthode qui ne peut empêcher la métaphysique occidentale de ressurgir au moindre processus de pensée. Il confie à la "déconstruction » la mission de surmonter ces aspects fondamentaux dont l'être ne parvient jamais à s'extraire totalement. Derrida utilise la métaphysique occidentale pour mieux s'en affranchir et cette tâche ambiguë s'étend aux domaines les plus divers. Le philosophe critique les réalisations industrielles de la science et de la technique, s'en prend à la planification rationnelle des groupes sociaux, à la standardisation des connaissances et de la 
production, à la foi dans le progrès rationaliste. Il remet en cause les aspects dogmatiques propres à tout système de pensée, la "déconstruction " pulvérisant les tentations d'absolu susceptibles de déconnecter la relation entre épreuve et vérité; mais elle entend aussi utiliser la refondation des valeurs pour former l'homme nouveau d'une démocratie future. Derrida constate la fragilité de nos régimes démocratiques face au déferlement de la terreur. Les Droits de l'Homme et l'idéal cosmopolite européen sont pour lui des héritages capables de déchiffrer ce qu'il y a de nouveau dans les désordres mondiaux, la République devant être critique à la fois contre l'identité et les discriminations. Le philosophe s'en prend aux idéologies politiques et pourfend le néolibéralisme; mais il estime que le totalitarisme soviétique a laissé subsister intact un idéal communiste. Le spectre de la révolution hanterait toujours la conscience occidentale et le socialisme scientifique mériterait une certaine réhabilitation. La "déconstruction»appliquée aux idéologies n'exclut donc aucune refondation communiste et la critique des concepts ne s'arrête pas au scepticisme d'une pensée affirmant que rien n'est jamais achevé. Derrida affectionne cette ambivalence des valeurs propre aux démarches qui mettent sans cesse la vérité à l'épreuve et en donnent une interprétation précaire. Il en résulte des dérives confondant fantasme et réalité dans un immense commentaire tournant indéfiniment sur lui-même comme une rhétorique casuistique; mais la méthode ne manque pas de réalisme ni d'actualité. Derrida reproche ainsi au structuralisme un ethnocentrisme incompatible avec l'ouverture des sociétés occidentales au multiculturalisme. Un processus d'universalisation doit transgresser les limites ethniques, géographiques et politiques. L'intérêt suscité par Derrida vient aussi de ses réflexions sur l'inconscient et le tragique de l'existence que nombre de ses adeptes préfèrent à une philosophie de la conscience ou du sujet.

2 La violence est constamment présente dans l'œuvre du philosophe de la "déconstruction" qui se plait à opposer la "violence fondatrice " à une "violence conservatrice » fondamentalement destructrice. Ce thème prend toute sa signification dans l'éducation, présentée comme une violence institutionnalisée si intimement liée à une "violence métaphysique» qu'elle en oublie le message éducatif. Alessandro Mariani n'est pas insensible à ce radicalisme hypercritique et il souhaite puiser aux sources mêmes de la connaissance l'élaboration de nouvelles finalités éducatives. Ce retour à une philosophie de l'éducation impliquerait une réforme du mode de penser et d'enseigner. L'expérience de la « déconstruction » critique par exemple la séparation traditionnelle entre l'enseignement considéré comme insertion d'une donnée externe et l'éducation conçue comme exploitation d'une donnée interne. L'auteur propose une philosophie de l'éducation qui fournirait les instruments nécessaires à la critique des institutions et des pratiques éducatives. Cette rationalité non dogmatique dialoguerait en permanence avec la pédagogie en lui fournissant les outils critiques indispensables pour lui éviter de s'enfermer dans des didactiques stériles inspirées des scientismes et des idéologies. Le chemin déjà parcouru est jalonné de révoltes contre la métaphysique fomentées par l'« école du soupçon » de la triade Marx, Nietzsche et Freud. Il a certes conduit à l'obscur enchevêtrement des sciences de l'éducation; mais il a permis une critique radicale de l'école et de la famille qui perpétuent, selon l'auteur, des liens de dépendance et le cloisonnement social. En rapprochant le texte de l'expérience, la « déconstruction » s'en prend à l'hégémonie d'une culture dogmatique relayée par les médias et elle permet d'élaborer un discours pédagogique orienté vers de nouvelles perspectives. Les concepts d'instruction et d'éducation s'affineraient au contact d'une 
réflexion philosophique assignant un rôle central à la formation ainsi qu'aux valeurs éthiques et sociales du savoir. Le maître doit respecter la démarche de la "déconstruction » dans ses enseignements. Dans cette perspective, les réactions des élèves deviennent essentielles ainsi que l'ambivalence des termes et des concepts. Il faut valoriser ce qui est marginal pour détruire les hiérarchies dominantes et empêcher d'en élaborer de nouvelles.

On ne peut que se réjouir d'une herméneutique des savoirs éducatifs préservant la pédagogie de toute théorisation. Elle se libère des pédagogismes asservis aux sciences et aux idéologies pour s'affirmer interprétative et non plus normative. Dans une société où règnent la «logorrhée " médiatique et le "triomphalisme économique ", le retour au texte peut être fondateur. Sans lui, nous rappelle l'auteur, l'idée n'est rien. Il est indéniable qu'une étude des textes négligeant la complexité des pratiques d'écriture et le caractère contingent des différences affichées par les acteurs peut conduire à l'élaboration de catégories artificielles; mais passer les documents et les témoignages au crible d'une méthode hypercritique conduit à une lecture maximaliste qui rend toute synthèse impossible. En s'affranchissant de l'enchantement des grandes narrations et de la métaphysique des fondements, la « déconstruction » entend libérer le corps pour en faire cet espace inaliénable qui détient la clé de l'être; mais en récusant la valeur formative du progrès du savoir, la "déconstruction " privilégie des connaissances permettant à l'individu de se remettre constamment en cause. Il en résulte un scepticisme qui génère des incertitudes sur le plan éthique.

Notre société occidentale moderne a rompu le cercle magique en repoussant le référentiel religieux aux marges de ses logiques d'action pour lui substituer la foi en une science triomphante qui ouvre le temps, l'espace, l'individu et la conscience aux grandes aventures de l'esprit et de la connaissance. La "déconstruction » figure en bonne place dans cette fuite de l'absolu qui conduit l'homme moderne à perdre le sens de l'existence. L'interdiction de croire en des valeurs suprêmes enferme l'individu rongé par le doute dans un univers d'angoisse qui confine à cette " hantise " toujours présente dans l'œuvre de Derrida. Le rejet de tout principe absolu condamne à l'abandon de l'idée fondamentale de rédemption. Elle prétend y remédier par le principe chrétien du don de soi et par l'enrichissement moral de l'amitié ; mais elle leur prête surtout les vertus d'une expérience sociale et néglige les effets de ce lien sur la conscience individuelle. L'auteur semble en être conscient lorsqu'il insiste sur l'intérêt du lien affectif unissant le maitre à ses disciples. La French theory a sans doute conquis les États-Unis au début des années 2000 ; mais on dit que les universités américaines en sont déjà revenues. Plus que ses réponses contestables, on retiendra ses interrogations sur la pensée occidentale et sur la construction culturelle et sociale du réel qui sont des questions fondamentales pour l'avenir. 